\title{
Isolation and Identification of Three Parvovirus Strains in Rac- coon Dogs from Liaoning Province, China
}

\author{
Xiaoqin Guo ${ }^{1}$, Zhuochen Li ${ }^{1}$, Tiantian $\mathrm{Lv}^{1}$, Haixu Cao ${ }^{1}$, Xin Du ${ }^{3}$, Zirui Li ${ }^{2, *}$ and Chuanfang Zhao ${ }^{1, *}$ \\ 1 Institute of Special Animal and Plant Sciences, Chinese Academy of Agricultural Sciences, Chang- \\ chun,130112 China \\ 2 Logistics Service Center of Chinese Academy of Agricultural Sciences, Beijing,100081 China \\ 3 Jilin Guanjie Biological Technology Co., LTD, Meihekou 135000 China \\ * Correspondence: lizirui@caas.cn, zhaochuanfang1213@163.com.
}

\begin{abstract}
To understand the epidemiological status of parvovirus (RDPV) in raccoon dogs, intestinal tissues of raccoon dogs in Liaoning Province of China were collected and evaluated. Three strains of raccoon dog parvovirus were successfully isolated from 12 intestinal tissues. Nine samples were positive for RDPV, with a positive rate of 75\%. The VP2 and NS1 genes of the viruses were cloned and subjected to sequencing for analysis. The nucleotide sequences of the VP2 gene showed 99.94\% similarity to the CPV-2a/Racoon dog/QHD/2/19(MT183665) strain, and the nucleotide sequences of the NS1 gene showed 99.75\% similarity to RDPV-DP1 NS1(MF996335) strain. The three isolates belonging to the CPV-2a cluster were further confirmed by amino acid sequence alignment and phylogenetic analysis. Our study enriched the epidemiological data of parvovirus in raccoon dogs in the investigating region, and the results will be helpful for future investigation of the variations and transmission of raccoon dog parvoviruses.
\end{abstract}

Keywords: Raccoon dog parvovirus; Epidemiology; VP2 gene; NS1 gene; Evolutionary analysis

\section{Introduction}

Racoon dog parvovirus (RDPV) is a member of the family Parvoviridae, genus Protoparvovirus, which has more than $98 \%$ genomic homology with canine parvovirus (CPV), feline panleukopenia virus (FPLV), mink enteritis virus (MEV), raccoon parvovirus (RPV), and blue fox parvovirus (BFPV) [1].

Carnivore parvoviruses can break through the interspecies barrier and gradually evolve from the same progenitor strain. After obtaining the ability of cross-species transmission, the virus is gradually evolved into species-specific [2-5]. In 1978, CPV-2 was first identified in dogs, which has been recognized by researchers as a way for FPLV to infect dogs with the help of intermediate hosts, such as foxes or raccoons[6]. RDPV was first detected in Heilongjiang Province in China in 1984. According to data in NCBI (National Center of Biotechnology Information), most of the raccoon dog parvovirus strains belong to the original CPV-2 subtype. In 2012, Kang et al. isolated a canine parvovirus strain LN10-1, which was a subtype between FPLV and CPV-2 subtype branches in the phylogenetic tree, indicating that LN10-1 strain may be in the middle state of evolution between FPLV and CPV [7]. In 2014, a CPV-2a mutant was isolated from Heilongiiang by Hu et al. [8], with a higher similarity in genetic evolution to that of the CPV-2, indicating that RDPV HLJ11-1 is in the middle state of FPLV and CPV evolution. In 2016, Lu and colleagues conducted a molecular epidemiological study of RDPV in Hebei Province and found that there were important non-synonymous mutations of several amino acid residues in the major capsid protein VP2, such as S27T, S297A, and I418T, indicating that the RDPV strains prevalent in China have been evolved to a certain extent [9].

Because of the frequent mutation rate of raccoon dog parvovirus in recent years, thus, continuous epidemiological surveillance of CPV-2 in time, space, and the host is undoubtedly an effective way to reveal its directed evolution driving force. At the same time, 
studies on natural (random) variations and pressure variations of the CPV-2 genome could also provide an effective example for us to explore the mechanisms underlying genetic variations of other viruses[10].

\section{Materials and Methods}

\subsection{Animals and specimens}

Raccoon dogs farmed in Liaoning Province exhibited symptoms of vomiting and diarrhea, which were suspected of RDPV infections. Thirty-one specimens of intestinal tissue and intestinal contents were taken from the sick or dead raccoon dogs, which were tested positive using colloidal gold test strips (Anjie, Quicking Biotech, Shanghai, China).

The specimens were fully ground, and diluted with PBS at a ratio of 1:5, followed by repeated freezing and thawing at $-80{ }^{\circ} \mathrm{C}$ for 3 times and centrifugation at $5,000 \mathrm{rpm}$ at $4{ }^{\circ} \mathrm{C}$ for $45 \mathrm{~min}$. The supernatant was taken and filtered with a $0.22 \mu \mathrm{m}$ filter, followed by the addition of double antibiotics (penicillin and streptomycin) to a final concentration of $100 \mathrm{U} / \mathrm{ml}$. After treatment overnight at $4{ }^{\circ} \mathrm{C}$, the supernatants were kept at $-80{ }^{\circ} \mathrm{C}$.

\subsection{Virus Isolation}

The supernatants of the grounded tissues were added to F81 cells, which were dissociated and resuspended in growth median (MEM with 10\% FCS) at a ratio of $10 \%$ $(2 \mathrm{ml} / 20 \mathrm{ml})$. Cells without inoculation were used as a blank control. The cells were cultured at $37{ }^{\circ} \mathrm{C}$ in a $5 \% \mathrm{CO}_{2}$ incubator and observed for cytopathic effects every day. When the cytopathic effects reached about $80 \%$, the cells were collected, and then frozen and thawed 3 times. After brief centrifugation, the supernatant was taken and used to infect the new cells as described. After three passages, the virus-containing supernatant was stored at $-80{ }^{\circ} \mathrm{C}$.

\subsection{Electron microscopy observation}

$200 \mathrm{ml}$ of virus-containing supernatant was centrifugated at $4{ }^{\circ} \mathrm{C} 10000 \mathrm{rpm}$ for 10 min. The cleaned supernatant was added $8 \%$ PEG6000 and $3 \% \mathrm{NaCl}$ and was stirred overnight at $4{ }^{\circ} \mathrm{C}$ to precipitate the virions. After centrifugation at $10000 \mathrm{rpm}$ for $90 \mathrm{mins}$ at 4 ${ }^{\circ} \mathrm{C}$, the pellet was resuspended with $1 \mathrm{ml} \mathrm{STE}$ buffer [11]. $10 \mathrm{uL}$ of the virus preparation was dropped on a fresh-keeping film, followed by clipping a carbon-coated copper net onto the virus droplet for $10 \mathrm{~min}$. Then, the viruses on the wet copper net were stained with $1 \%$ phosphotungstic acid solution, followed by observation of the morphological characteristics of the virus under an electron microscope (Hitachi TEM system).

\subsection{Amplification, cloning, and sequencing of the VP2 and NS1 genes}

Viral DNA was extracted using the DNA rapid extraction kit (TRANSGEN Biotech, Beijing, China) according to the manufacturer's instructions. Regarding the VP2 and NS1 gene sequences of the RDPV strain deposited in GenBank (EU659117), primers were designed using Primer5.0 software and used to amplify the targeted fragments (Table 1).

Table 1. Primers and sequences .

\begin{tabular}{cc}
\hline Primer & Sequence $\left(5^{\prime} \rightarrow 3^{\prime}\right)$ \\
\hline VP2-F & ATGAGTGATGGAGCAGTTCAAC \\
VP2-R & TTAATATAATTTTCTAGGTGCTAGTTGAG \\
NS1-F & ATGTCTGGCAACCAGTAT \\
NS1-R & TTAATCCAAGTCGTCTCGAAAA \\
\hline
\end{tabular}

The PCR reaction system was set up in $50 \mu \mathrm{L}$ as follows: template $2 \mu \mathrm{L}, 2 \times$ LA Taq2 25 $\mu \mathrm{L}, 1 \mathrm{ul}$ for each of the upstream and downstream primers. PCR reactions were at $55{ }^{\circ} \mathrm{C}$ for $30 \mathrm{~s}$ and $72{ }^{\circ} \mathrm{C}$ for $10 \mathrm{mins}$ for 30 cycles. The PCR products were run on $1 \%$ agarose gel electrophoresis.

The DNA bands at the expected size of $1755 \mathrm{~kb}$ (VP2) and $2007 \mathrm{~kb}$ (NS1) were excised and extracted, followed by ligation to PEasy-T1 clone vector (TRANSGEN Biotech, 
Beijing, China), transformation into DH-5 $\alpha$ competent cells(TRANSGEN Biotech, Beijing, China) and culture in LB petri dish containing ampicillin $(100 \mathrm{mg} / \mathrm{ml})$ at $37{ }^{\circ} \mathrm{C}$ overnight. On the next day, bacterial conies were picked up for amplification. Plasmids were extracted from the culture and identified by restriction digestion with Hind III and Xho I. The positive ones were subjected to sequencing at Jilin Kumei Biotechnology Co. Ltd.

\subsection{VP2 and NS1 Phylogenetic analysis}

We blasted the complete VP2 and NS1 sequences obtained in this study and selected the nucleotide sequences with high similarity to their gene sequences for homology analysis. The MegALign program in DNAstar was used to analyze the VP2 gene sequences of the new isolates and other carnivore parvovirus VP2 gene sequences deposited in GenBank. We used Mega7.0 software to construct a phylogenetic tree with maximum similarity proximity (Maximum Likelihood) algorithm.

Table 2. comparison of key amino acid sites between isolated strains and reference strains. 


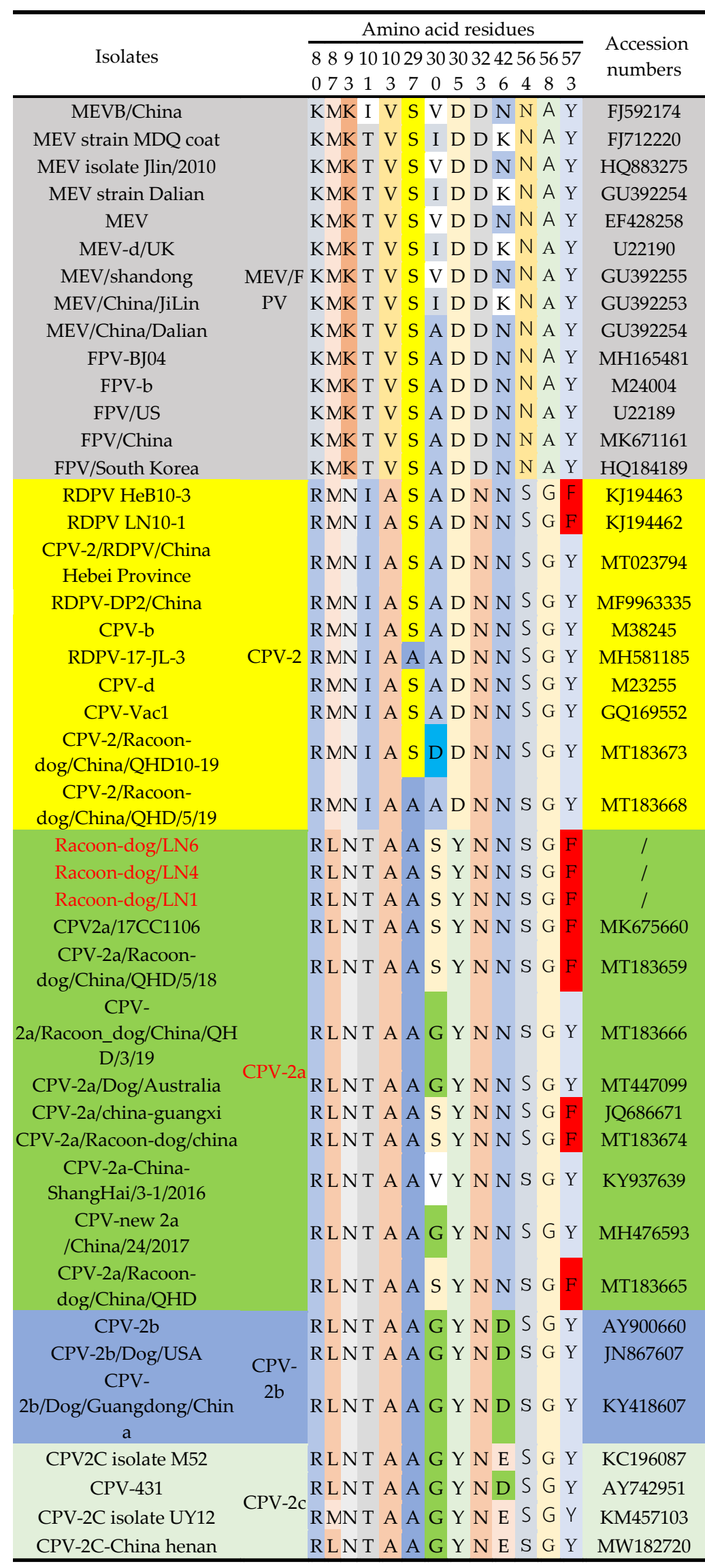


Five healthy raccoon dogs whose parvovirus antibody was less than 1:4 were selected (hemagglutination inhibition test). Three raccoon dogs were fed with $20 \mathrm{ml}$ of virus-containing supernatant of passage 3, the rest two raccoon dogs were fed with $20 \mathrm{ml}$ of MEM as a control, and they were fed in isolation. After infection, the mental state, diet, and defecation of raccoon dogs were observed every day. The dead ones during the infection were dissected in time, and the pathological changes of tissues and organs were examined. Tissues and organs, such as lung, heart, liver, stomach, spleen, kidney, intestine, and mesenteric lymph nodes were fixed with $10 \%$ formalin and HE stained for the observation of the pathology.

\section{Results}

\subsection{Virus isolation}

We observed the F81 cells inoculated with RDPV-LN strain became swollen and showed typical pathological changes, while the control cells had a clear outline and uniform shape and size (Fig. 1).

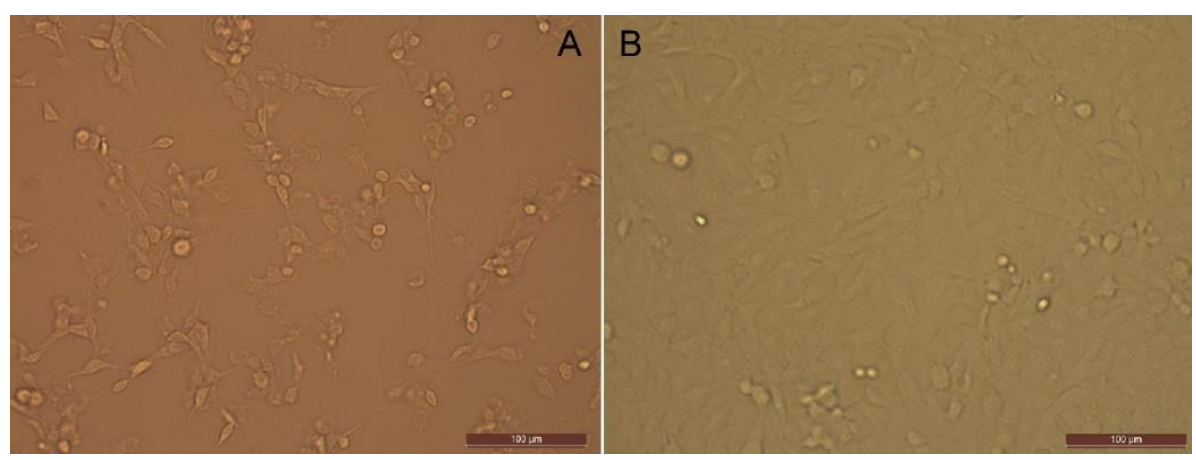

Figure 1. Morphology of infected and uninfected F81 cells. (A) F81 cells infected with an isolate. (B) control F81 cells.

\subsection{Electron microscopy observation}

We observed virus-like particles under the electron microscope, which had a diameter of about $22 \mathrm{~nm}$ without an envelope and exhibited an icosahedral symmetrical structure (Fig. 2), typical features of parvovirus.
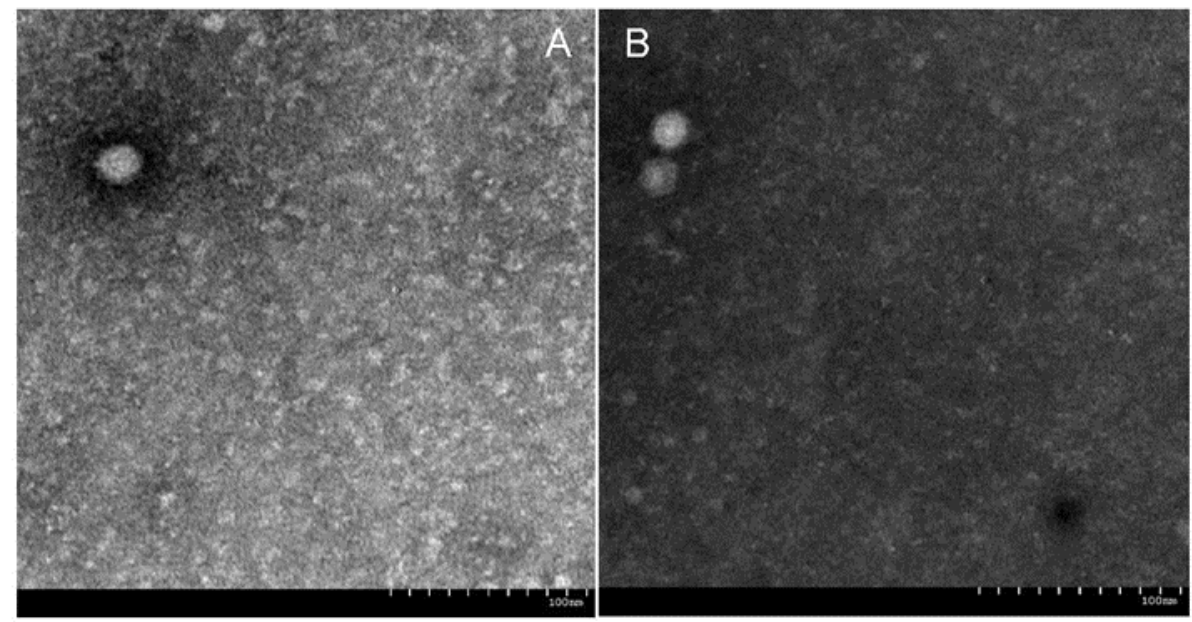

Figure 2. The morphology of virus particles under the electron microscope.

\subsection{Raccoon dog infection experiments}

Immunostaining of the pathological sections showed intestinal villous epithelial defect, intestinal villus atrophy, necrotic cell exfoliation, protein-like secretion, and neutrophil mixed mass in intestinal gland and crypt, and obvious neutrophil and eosinophil 
infiltration in lamina propria. There was a partial defect of the villous epithelium of the large intestine, and massive necrotic cells, protein-like secretion, and neutrophil mixed formation were also observed in the intestinal gland, which was more severe than that of the small intestine. In addition, neutrophil infiltration and eosinophil infiltration were observed in the lamina propria, which, however, were not as significant as those in the small intestine.

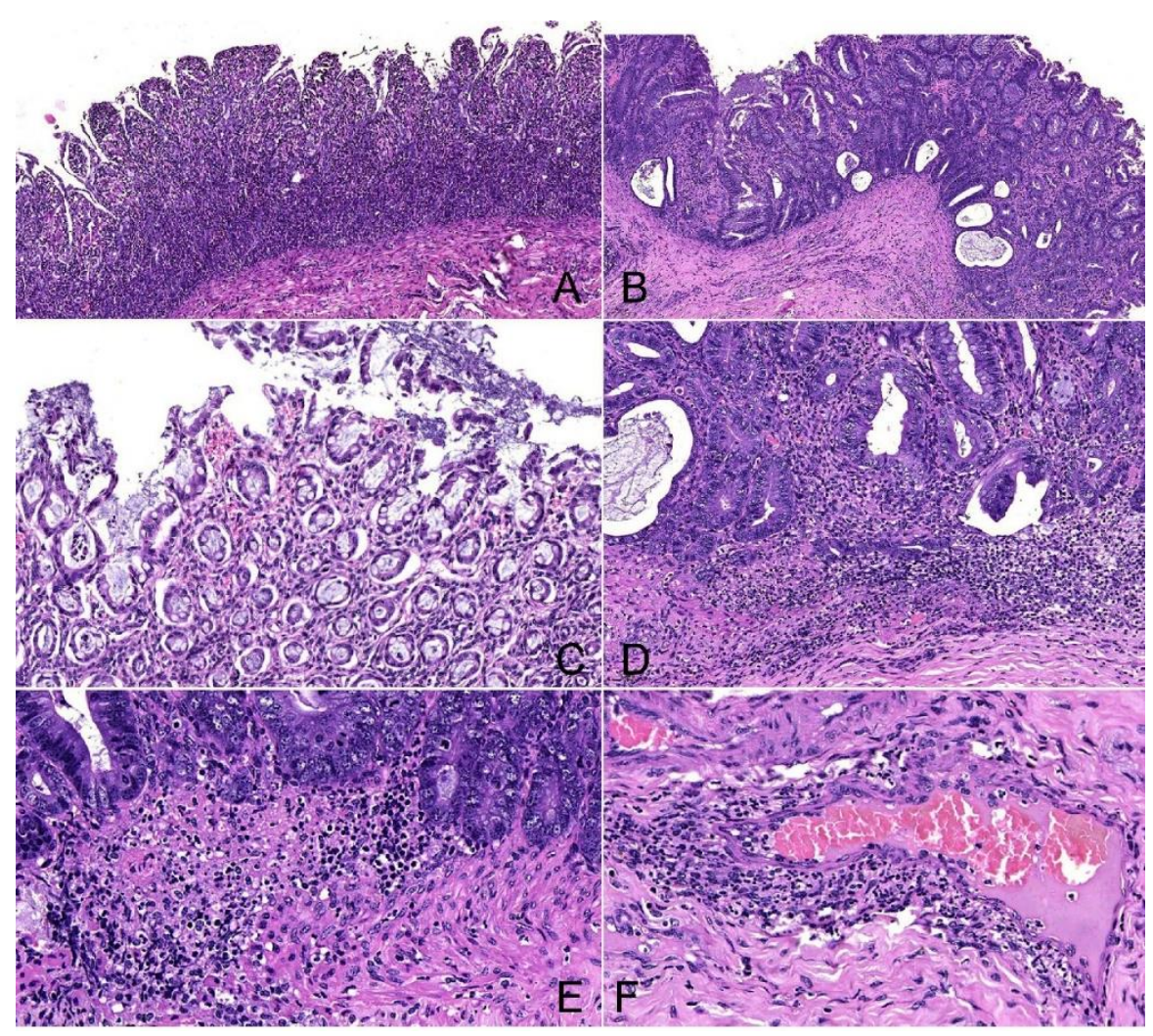

Figure 3. Immunohistochemistry staining of pathological sections of intestinal tissues of animals infected with RDPV. (A) Small intestinal villous epithelium exfoliated, lamina propria exposed, a large number of inflammatory cells infiltrated in the lamina propria of the intestinal mucosa. (B) large intestinal gland dilated, mucus-like secretion or protein-like secretion in the glandular cavity, mixed with exfoliated cell component. (C) Large intestinal mucosal epithelium exfoliated, lamina propria hyperemia, and hemorrhage. (D) Severe central granulocyte infiltration. From the deep layer of the lamina propria of the large intestine to the submucosa. (E) Necrosis of the submucosa of the large intestine and perivasculitis of the large intestine. 


\subsection{Phylogenetic analyses of VP2 and NS1 gene sequences}

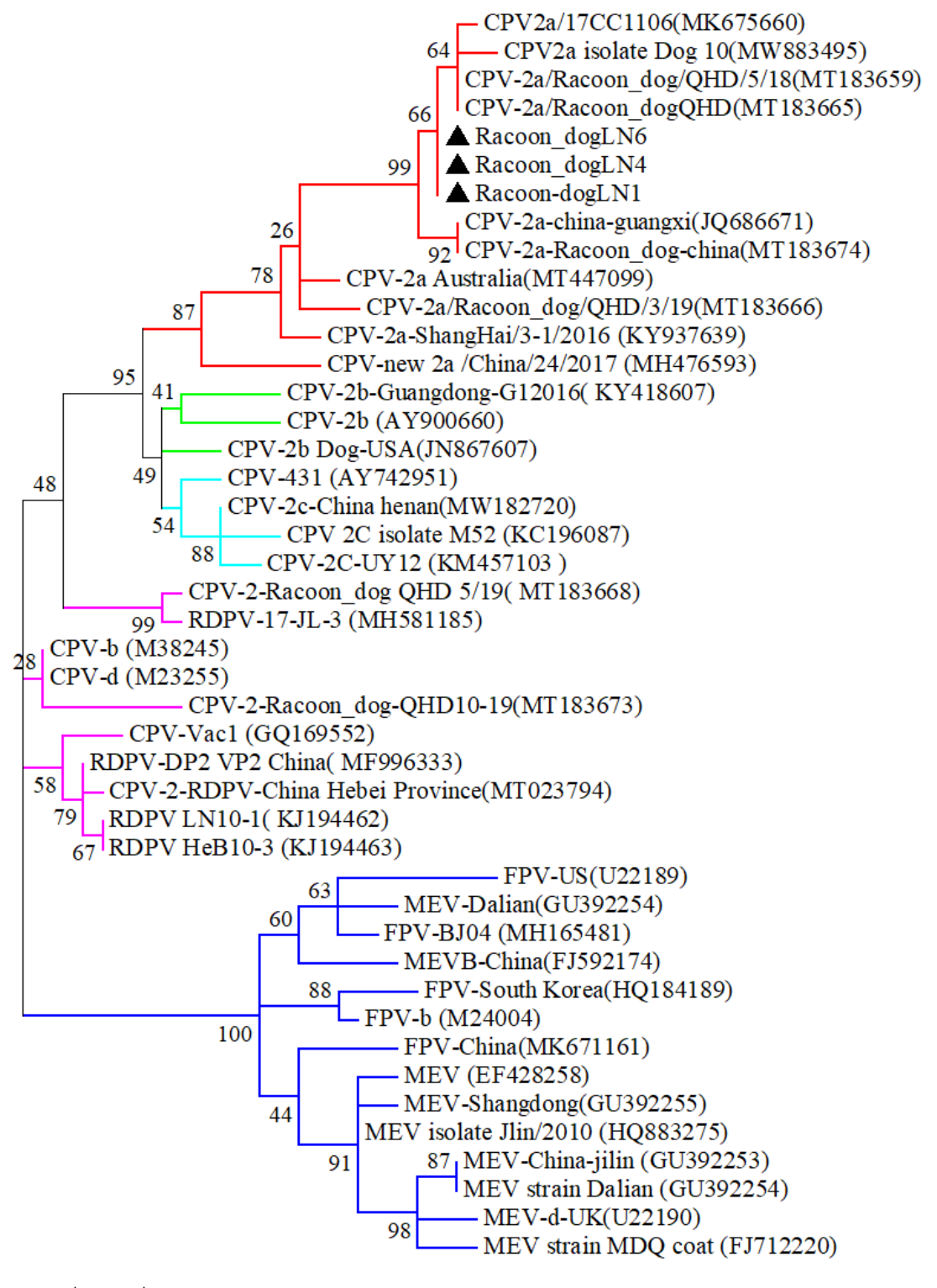

Figure 4. Phylogenetic analysis of the VP2 gene sequences of RDPV and related parvoviruses. 


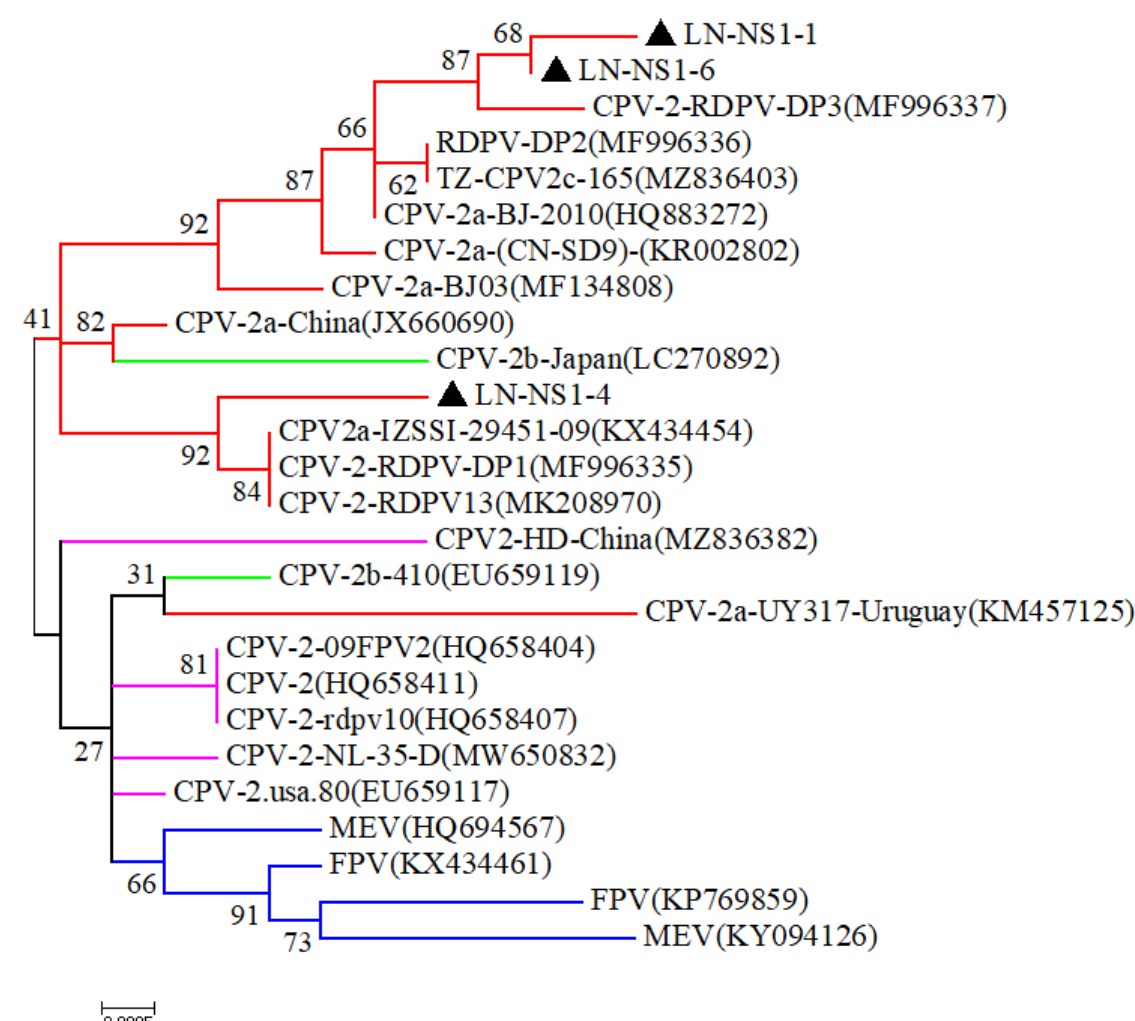

CPV-2a

CPV $-2 b$

Virus strains isolated

CPV-2

$\mathrm{MEV} / \mathrm{FPV}$

Figure 5. Phylogenetic analysis of NS1gene sequences of RDPV and related parvoviruses.

\section{Discussion}

Parvovirus is a small nonenveloped virus of $25 \mathrm{~nm}$ in diameter with a linear singlestranded DNA genome of Approx. $5 \mathrm{~kb}$ with hairpin structures at both ends. The virus has two main open reading frames, one encodes two non-structural proteins (NS1 and NS2), and the other encodes capsid proteins VP1 and VP2 [12]. Among them, VP2 protein accounts for $90 \%$ of the virus capsid and is the most important protective antigen, which is closely related to the pathogenicity and virulence of the virus. VP2 protein has several key amino acid sites that can change the antigenicity, host range, and tissue tropism of the virus. The amino acids at positions $426^{\text {th }}$ and $297^{\text {th }}$ are the key factors for the differentiation of CPV-2 mutants (CPV-2a, CPV-2b, NewCPV-2a, NewCPV-2b, and CPV2c) [13]. When CPV-2 evolved to CPV-2a, amino acid residue 87 of the VP2 was changed from methionine $(\mathrm{M})$ to leucine (L), residue 300 from alanine (A) to glycine $(\mathrm{G})$, and residue 305 from aspartic acid (D) to tyrosine (Y) [14-17]. The three RDPV strains isolated in our study were in the same subclade as the strain MT183665 isolated from Qinhuangdao, Hebei Province in 2019, and were clustered into CPV-2a clade. According to their key amino acid sites, they were identified as CPV-2a type. Compared with the evolutionary RDPV strains, such as KJ194462, synonymous substitution has taken place at M87L, S297A, D305Y, A300S, and Y573F. Studies have shown that S297A mutation takes place under strong selection pressure, which may be related to local adaptability [18]. When the mutation at aa297 occurs, VP2 is expected to produce an extra small peptide, but the specific function is not clear [19]. aa300 is located at the tip of the CPV triploid cusp and plays an important role in the interaction between CPV and host transferrin receptor TfR. Mutation at aa300 may cause changes in the host range of CPV. It has been found that most raccoon dog parvovirus strains contain A at aa300 [20,21]. In recent years, the substitution of A300D has been found constantly, and the mutation of A300S suggests that this locus may be variable 
in RDPV $[13,22]$. Whether the mutation of Y573F will cause the change of protein function needs to be further verified. These amino acid changes in VP2 are sufficient for host transfer and adaptation, thus most studies have focused on the evolution of the VP2 gene, whereas studies on NS gene evolution are much less [14]. NS1 protein has the function of ATP enzyme and helicase, regulates the expression of other proteins, and is closely related to viral DNA replication, transcription and induction of cell apoptosis [23, 24]. The NS1 genes of the three RDPV isolates in our study were also clustered into CPV-2a clades. Overall, these results show that the epidemic strains of RDPV in Liaoning area of China have evolved to a certain extent.

The significance of this study is that the established vaccine candidates will provide the basis for the development of parvovirus vaccines in dogs and raccoon dogs. The study on the mechanism of CPV infection in dogs can provide early warning for the prevention and control of parvovirus disease in dogs and raccoon dogs in the future. The elucidation of the mechanism of CPV cross-species transmission can provide a theoretical basis for the prevention of potential public health security risks caused by CPV cross-species transmission in China.

Ethics approval and consent to participate: The animal experiment was examined by the Experimental Animal Management and Ethics Committee of The Institute of Special Animal and Plant Sciences, Chinese Academy of Agricultural Sciences (Approved ID:ISAPSAEC-2021-61).

Author Contributions: Conceptualization, Writing-original draft preparation, Xiaoqin Guo; Investigation, Zhuochen Li, Xiaoqin Guo; Resources, Tiantian Lv, Haixu Cao, Xin Du; Supervision, Zirui Li, Chuanfang Zhao. All authors have read and agreed to the published version of the manuscript.

Funding: This work was financially supported by Jilin Province Science and Technology Department key research and development project(20200402049NC). The funders had no role in the study design, data collection, and analysis, decision to publish, or preparation of the manuscript.

Conflicts of Interest: The authors declare that they have no competing interests. All authors have approved the final version of the manuscript.

Availability of data and materials: All data generated or analyzed during this study are included in this published article.

Informed Consent Statement: Not applicable.

\section{References}

1. Cotmore, S. F.; Agbandje-McKenna, M.; Chiorini, J. A.; Mukha, D. V.; Pintel, D. J.; Qiu, J.; Soderlund-Venermo, M.; Tattersall, P.; Tijssen, P.; Gatherer, D.; Davison, A. J., The family Parvoviridae. Arch Virol 2014, 159, (5), 1239-1247.

2. Kaelber, J. T.; Demogines, A.; Harbison, C. E.; Allison, A. B.; Goodman, L. B.; Ortega, A. N.; Sawyer, S. L.; Parrish, C. R., Evolutionary reconstructions of the transferrin receptor of Caniforms supports canine parvovirus being a re-emerged and not a novel pathogen in dogs. PLoS Pathog 2012, 8, (5), e1002666.

3. Allison, A. B.; Kohler, D. J.; Ortega, A.; Hoover, E. A.; Grove, D. M.; Holmes, E. C.; Parrish, C. R., Host-specific parvovirus evolution in nature is recapitulated by in vitro adaptation to different carnivore species. PLoS Pathog 2014, 10, (11), e1004475.

4. Allison, A. B.; Kohler, D. J.; Fox, K. A.; Brown, J. D.; Gerhold, R. W.; Shearn-Bochsler, V. I.; Dubovi, E. J.; Parrish, C. R.; Holmes, E. C., Frequent cross-species transmission of parvoviruses among diverse carnivore hosts. J Virol 2013, 87, (4), 23422347.

5. LU, R. G.; LIU, W. Q.; YAN, X. J., Advance in Mechanisms of Canine Parvovirus Interaction with Host and Cross-species Transmission. Progress in Veterinary Medicine 2020, 41, (11), 113-116.

6. Truyen, U., Evolution of canine parvovirus--a need for new vaccines? Vet Microbiol 2006, 117, (1), 9-13.

7. KANG, H. T. Isolation and Immunogenic Evaluation of Raccoon Dog Parvovirus. 硕士, Chinese Academy of Agricultural Sciences, 2012.

8. HUYAN, L. H.; QIAN, A. D.; ZHANG, H. L.; al, e., Isolation and identification of the HLJ11-1 strain of raccoon dog parvovirus and the sequence analysis of the VP2 gene. Heilongjiang Animal Science and Veterinary Medicine 2014, (17), 11-16+226.

9. LU, R. G.; YAN, X. J., A new type of CPV-2 broke out in the Chinese opulation. Special wild economic animal and plant research 2019, 41, (01), 103.

10. Zhao, J.; Yan, X.; Wu, W., [Origin and evolution of canine parvovirus--a review]. Wei Sheng Wu Xue Bao 2011, 51, (7), 869875. 
11. ZHAO, G. X. Establishment and application of two rapid detection methods for canine parvovirus enteritis. 硕士, Jilin University, 2015.

12. Parrish, C. R., Host range relationships and the evolution of canine parvovirus. Vet Microbiol 1999, 69, (1-2), $29-40$.

13. Allison, A. B.; Organtini, L. J.; Zhang, S.; Hafenstein, S. L.; Holmes, E. C.; Parrish, C. R., Single Mutations in the VP2 300 Loop Region of the Three-Fold Spike of the Carnivore Parvovirus Capsid Can Determine Host Range. J Virol 2016, 90, (2), 753-767.

14. Hueffer, K.; Parker, J. S.; Weichert, W. S.; Geisel, R. E.; Sgro, J. Y.; Parrish, C. R., The natural host range shift and subsequent evolution of canine parvovirus resulted from virus-specific binding to the canine transferrin receptor. J Virol 2003, 77, (3), 1718-1726.

15. Ikeda, Y.; Mochizuki, M.; Naito, R.; Nakamura, K.; Miyazawa, T.; Mikami, T.; Takahashi, E., Predominance of canine parvovirus $(\mathrm{CPV})$ in unvaccinated cat populations and emergence of new antigenic types of CPVs in cats. Virology 2000, 278, (1), 13-19.

16. Parrish, C. R.; Aquadro, C. F.; Strassheim, M. L.; Evermann, J. F.; Sgro, J. Y.; Mohammed, H. O., Rapid antigenic-type replacement and DNA sequence evolution of canine parvovirus. J Virol 1991, 65, (12), 6544-6552.

17. Truyen, U.; Evermann, J. F.; Vieler, E.; Parrish, C. R., Evolution of canine parvovirus involved loss and gain of feline host range. Virology 1996, 215, (2), 186-189.

18. Pereira, C. A.; Leal, E. S.; Durigon, E. L., Selective regimen shift and demographic growth increase associated with the emergence of high-fitness variants of canine parvovirus. Infect Genet Evol 2007, 7, (3), 399-409.

19. Nelson, C. D.; Minkkinen, E.; Bergkvist, M.; Hoelzer, K.; Fisher, M.; Bothner, B.; Parrish, C. R., Detecting small changes and additional peptides in the canine parvovirus capsid structure. J Virol 2008, 82, (21), 10397-10407.

20. Son, K.; Lee, S. M.; Kim, Y.; Kim, Y. K.; Lee, S. Y.; Jheong, W. H.; Oem, J. K., Genetic Characterization of Canine Parvovirus Type 2 Detected in Wild Raccoon Dogs ( Nyctereutes procyonoides) in the Republic of Korea. J Wildl Dis 2019, 55, (2), 512515.

21. Jia-Yu, Y.; Qian, Z.; Fei-Fei, D.; Chuan-Jie, T.; Hui, P.; Yuan-Yuan, S.; Yong-Feng, Z.; Jian-Li, W.; Jiang, S.; Zhi-Jing, X., Emergence of novel canine parvovirus type 2 and its pathogenesis in raccoon dogs. Vet Microbiol 2018, 216, 7-12. Lu, R.; Yu, Y.; Zhu, X.; Shi, Q.; Wang, Y.; Wang, J.; Lv, S.; Shi, N.; Liu, H.; Deng, X.; Lian, S.; Yan, M.; Zhao, H.; Hu, B.; Liu, W.; Yan, X., Molecular characteristics of the capsid protein VP2 gene of canine parvovirus type 2 amplified from raccoon dogs in Hebei province, China. Arch Virol 2020, 165, (11), 2453-2459.

23. Saxena, L.; Kumar, G. R.; Saxena, S.; Chaturvedi, U.; Sahoo, A. P.; Singh, L. V.; Santra, L.; Palia, S. K.; Desai, G. S.; Tiw ari, A. K., Apoptosis induced by NS1 gene of Canine Parvovirus-2 is caspase dependent and p53 independent. Virus Res 2013, 173, (2), 426-430.

24. Gilbert, L.; Välilehto, O.; Kirjavainen, S.; Tikka, P. J.; Mellett, M.; Käpylä, P.; Oker-Blom, C.; Vuento, M., Expression and subcellular targeting of canine parvovirus capsid proteins in baculovirus-transduced NLFK cells. FEBS Lett 2005, 579, (2), 385-392. 